\title{
Fístula colecistocólica: una causa poco frecuente de hemorragia digestiva baja
}

\author{
Cholecistocolic fistula: an uncommon cause \\ of lower gastrointestinal bleeding
}

\author{
M. Ostiz ${ }^{1}$, J.J. Vila ${ }^{1}$, J.L. García Sanchotena ${ }^{2}$, S. Ostiz ${ }^{2}$, I. Alaez ${ }^{3}$
}

\section{RESUMEN}

La fístula colecistocólica es un tipo poco común de fístula bilioentérica que tiene una presentación clínica variable y que generalmente aparece como complicación de la enfermedad litiásica biliar. Puede manifestarse en forma de dolor abdominal, náuseas, pérdida de peso, diarrea con o sin esteatorrea asociada, clínica dispéptica y más raramente como hemorragia digestiva baja, colangitis de repetición o incluso como un ileo biliar. Las técnicas más útiles para el diagnóstico son el TC abdominal, los estudios baritados y la colangiopancreatografía retrógrada endoscópica (CPRE).

Describimos el caso de una mujer de edad avanzada, pluripatológica, con fístula colecistocólica, que se presentó en forma de hemorragia digestiva baja. Fue estudiada mediante colonoscopia, TC abdominal y enema de bario. Durante el ingreso requirió transfusión de 4 concentrados de hematíes, desestimándose el tratamiento invasivo debido a su comorbilidad de base. Evolucionó favorablemente, con resolución espontanea del cuadro. Dos meses después permanecía asintomática.

Palabras clave. Fístula colecistocólica. Hemorragia digestiva baja.

\begin{abstract}
Cholecystocolic fistula is an uncommon biliaryenteric fistula with a variable clinical presentation that usually appears as a rare complication of gallstone disease. It can present with abdominal pain, nausea, weight loss, diarrhoea with or without associated steatorrhea, and dyspeptic symptoms. Rare cases have been reported with lower gastrointestinal haemorrhage and even with a gallstone ileous. The most useful techniques for diagnosis are CT, barium studies, and ERCP.

We report a case of a cholecistocolic fistula in an eldery woman with multiple medical comorbidities that presented as lower gastrointestinal bleeding. She was explored with colonoscopy, abdominal CT and barium enema. She required a total of 4 units of whole blood and because of her comorbidities a decision was made not to proceed with invasive treatment. She had a good evolution and was asymptomatic two months later.
\end{abstract}

Key words. Cholecystocolic fistula. Lower gastrointestinal haemorrhage.
1. Servicio de Aparato Digestivo. Complejo Hospitalario de Navarra. Pamplona.

2. Servicio de Radiología. Complejo Hospitalario de Navarra. Pamplona.

3. Servicio de Medicina Interna. Clínica Ubarmin. Pamplona.

Recepción: 29 de marzo de 2012

Aceptación provisional: 10 de mayo de 2012

Aceptación definitiva: 14 de mayo de 2012
Correspondencia:

Miriam Ostiz Llanos

Aparato Digestivo

Hospital Reina Sofía

Carretera Tarazona km 3

31500. Tudela. Navarra (España)

E-mail:miriam.ostiz@gmail.com 


\section{INTRODUCCIÓN}

La fístula colecistocólica es un tipo poco frecuente de fístula bilioentérica que tiene una presentación clínica variable y que aparece en la mayoría de los casos como una rara complicación de la enfermedad litiásica biliar. Se ha descrito una mayor incidencia en personas de edad avanzada y con comorbilidad asociada ${ }^{1}$.

Puede manifestarse en forma de dolor abdominal, nauseas, pérdida de peso, diarrea con o sin esteatorrea asociada, clínica dispéptica y más raramente como hemorragia digestiva baja, colangitis de repetición o incluso como un ileo biliar. Debido a su baja incidencia y a la variabilidad de síntomas con los que puede presentarse es una patología que muchas veces los clínicos no suelen tener en cuenta en los diagnósticos diferenciales de inicio, lo que puede demorar el tratamiento.

La edad avanzada, la enfermedad litiásica biliar, un cuadro de diarrea de larga evolución o la presencia de neumobilia en una prueba de imagen deberían alertarnos siempre sobre esta posibilidad.

\section{CASO CLÍNICO}

Presentamos el caso de una mujer de 78 años, con múltiples antecedentes entre los que destacan asma bronquial, obesidad mórbida, síndrome de apnea obstructiva del sueño, insuficiencia renal crónica con anemia e hiperparatiroidismo secundarios, adenocarcinoma de mama intervenido y tratado con QT y RT, valvulopatía mitral e insuficiencia cardiaca, fibrilación auricular, HTA, DM tipo 2, glaucoma bilateral, colelitiasis y colecistitis crónica.

Ingresa por cuadro de hematoquecia de 4 días de evolución, sin fiebre, dolor abdominal, ni cambios en el ritmo intestinal asociados y refiriendo un marcado empeoramiento de su disnea habitual.

En la exploración física destacaba una molestia difusa a la palpación en hipocondrio derecho, y analíticamente presentaba anemia con $\mathrm{Hb}$ de $7,3 \mathrm{~g} / \mathrm{dl}$ (N: 12-16), fibrinógeno de $727 \mathrm{mg} /$ dl (N:150-400), hiperglucemia e insuficiencia renal ya conocida con cifra de urea de $148 \mathrm{mg} / \mathrm{dl} \mathrm{y}$ creatinina de $2,1 \mathrm{mg} / \mathrm{dl}$, ambas cifras similares a las de analíticas previas.

Se le realizó colonoscopia en la que se apreciaba una imagen de doble luz a nivel del ángulo hepático del colon, presentando además de la luz natural del colon una segunda luz de menor calibre, por la que salía un contenido de aspecto bilioso (Fig. 1).

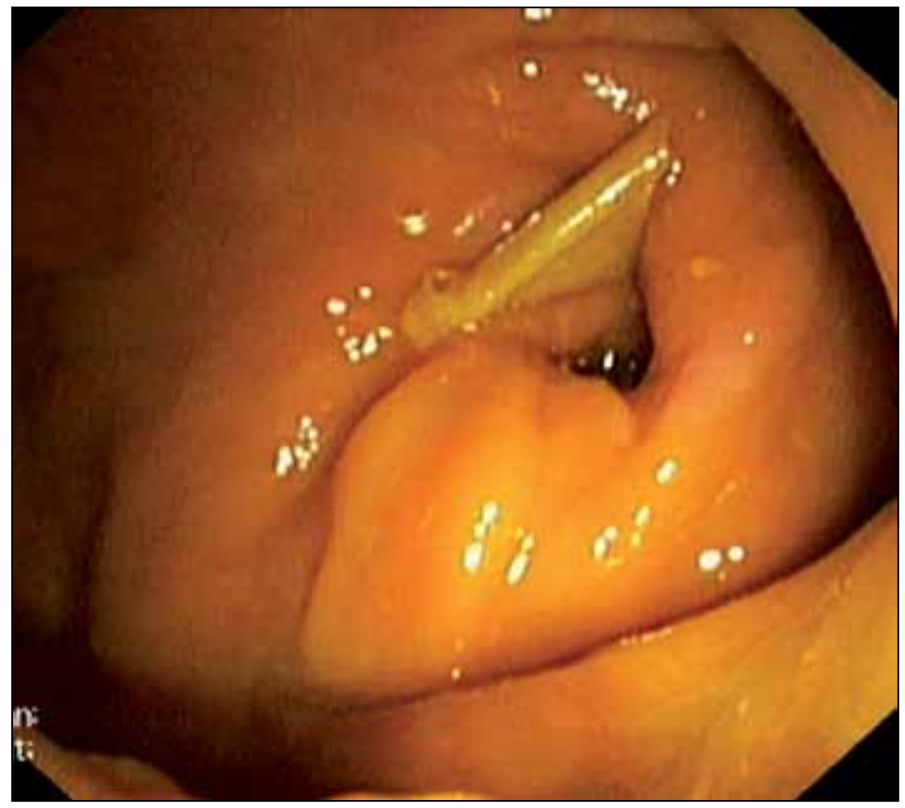

Figura 1. Trayecto fistuloso a nivel de ángulo hepático, con salida de contenido bilioso hacia la luz del colon. 
Ante estos hallazgos se solicitó un TC abdominal, que mostraba aerobilia, y una vesícula escleroatrófica en íntimo contacto con el ángulo

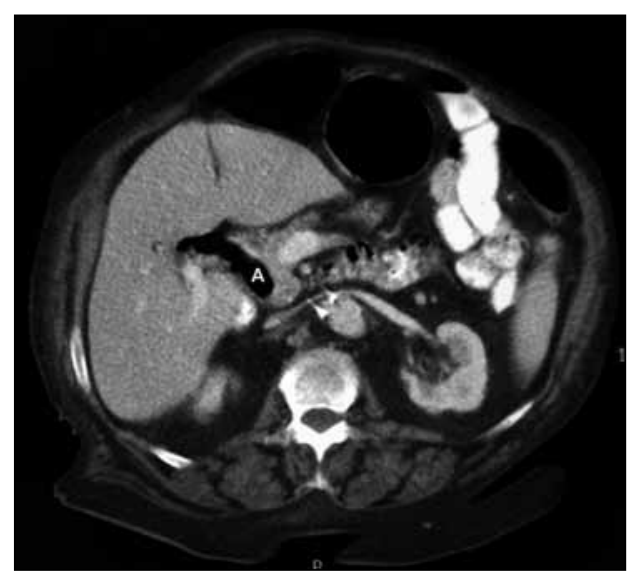

Figura 2a. Aerobilia (A) en TC abdominal.

Se decidió completar el estudio con un enema opaco en el que finalmente se objetivó la presencia de un trayecto fistuloso a nivel de ángulo hepático del colon, de un calibre aproximado de hepático del colon, cuya pared aparecía engrosada, pero sin alteración de la grasa subyacente (Figs. 2a y 2b).

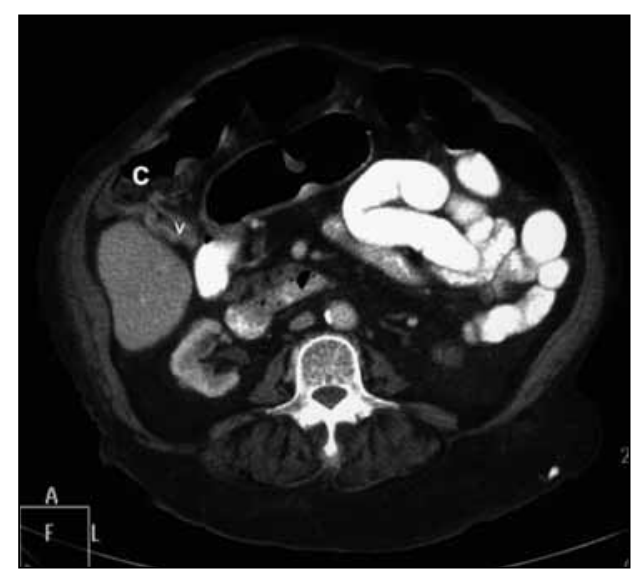

Figura 2b. Ángulo hepático del colon (C) y vesícula biliar (v) contraída sin contenido líquido en su interior y que presenta una pequeña burbuja gaseosa.

unos $6 \mathrm{~mm}$, que parecía comunicar con el colédoco, obteniéndose posteriormente relleno de la vía biliar intrahepática (Fig. 3).

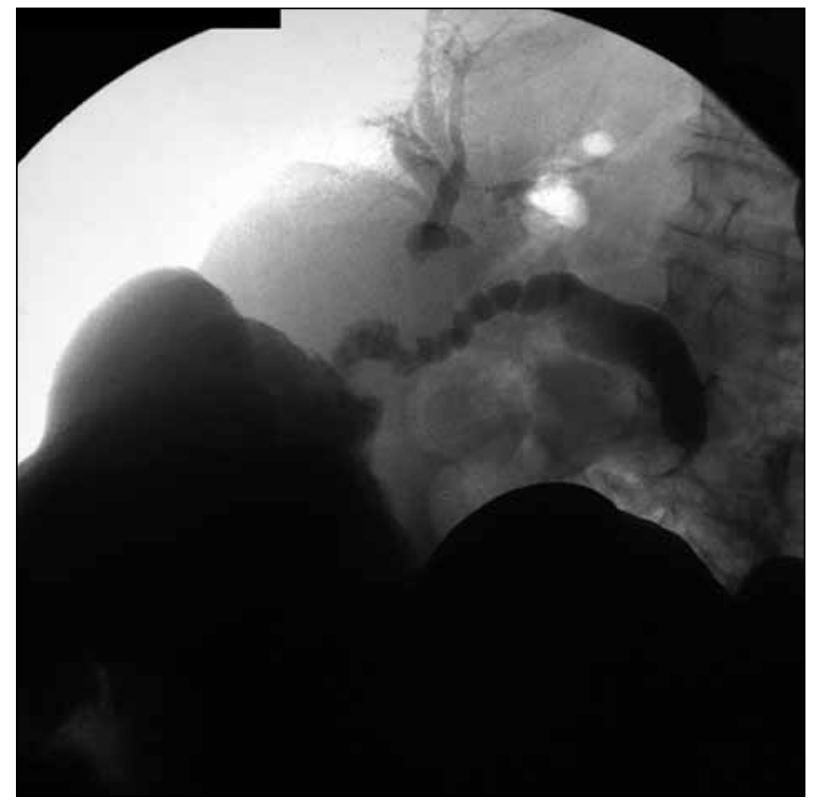

Figura 3. Paso de contraste desde colon rellenando el conducto cístico, el colédoco y parte de la vía biliar intrahepática. 
El diagnóstico final fue de colecistitis crónica con fístula biliar interna a ángulo hepático del colon y hemorragia digestiva baja secundaria.

Durante su ingreso precisó transfusión de 4 concentrados de hematíes, permaneciendo hemodinámicamente estable y afebril, sin presentar diarrea ni nuevos episodios de hematoquecia. Dada la pluripatología de base de la paciente y la buena evolución del cuadro agudo se decidió un manejo conservador, descartando intervención quirúrgica y CPRE terapeútica. Evolucionó favorablemente y fue dada de alta sin incidencias. Continuaba asintomática dos meses después del alta aunque falleció un tiempo después por patología cardiorrespiratoria.

\section{DISCUSIÓN}

La fistula colecistocólica es un tipo poco común de fístula bilio-entérica interna que se observa con más frecuencia en pacientes de edad avanzada y con comorbilidad asociada.

Aparece mayoritariamente como complicación de la enfermedad litiásica biliar, estando asociada a colelitiasis en un $90 \%$ de casos y a colecistitis crónica en un $12 \%$. En estos casos la inflamación puede favorecer la adhesión de la vesícula a la pared intestinal y esto, sumado a la presión que puede ejercer una litiasis puede ocasionar necrosis tisular y formación de fístulas. La comunicación más frecuente es la fístula colecistoduodenal que constituye el $70 \%$ de este tipo de fístulas, seguida por la fístula colecistocólica (10-20\%) y más raramente las fístulas coledocoduodenal y colecistogástrica ${ }^{2}$.

Aunque la patología biliar es la etiología principal de esta entidad, este tipo de fístulas pueden estar también asociadas a enfermedad inflamatoria intestinal, enfermedad diverticular, traumatismo abdominal, y neoplasias de colon, via biliar o páncreas $^{3-5}$.

Pueden ser asintomáticas, y detectarse de forma casual durante una cirugía (se ha descrito una incidencia del $0,5 \%$ en pacientes sometidos a colecistectomía) ${ }^{6}$, o presentarse en forma de dolor abdominal, náuseas, síntomas dispépticos y cuadro diarreico con esteatorrea en mayor o menor grado. Esta última presentación es relativamente frecuente y se debe a que la fistula altera la circulación enterohepática de los ácidos biliares, llevando a su malabsorción con la consiguiente esteatorrea. Así mismo estos ácidos biliares cuando llegan al colon irritan la mucosa provocando diarrea secretora, con importante pérdida de agua y electrolitos?

Otras presentaciones menos frecuentes de esta entidad son la hemorragia digestiva baja ${ }^{8-11}$, como en el caso que aquí se describe, colangitis de repetición o más raramente como un ileo biliar ${ }^{12}$.

Las técnicas diagnósticas más útiles en esta patología son el TC abdominal, los estudios baritados y la CPRE. La colonoscopia no siempre detecta la fístula, aunque en nuestro caso esta exploración sí fue diagnóstica.

En los casos sintomáticos el tratamiento de elección es quirúrgico, mediante colecistectomía electiva y reparación del trayecto fistuloso, que puede realizarse por cirugía abierta o por laparoscopia. En pacientes no quirúrgicos la CPRE está demostrando ser una técnica segura y poco invasiva, que permite la colocación de stents y la realización de esfinterotomía endoscópica, que ayuda a disminuir la presión biliar, favoreciendo así la obliteración espontánea del trayecto fistuloso ${ }^{13-15}$. En los casos que se presentan con diarrea, la resincolestiramina puede ayudar a controlar los síntomas mientras se está a la espera del tratamiento definitivo. Así mismo decir que también se han descrito casos de resolución espontánea, aunque con menor frecuencia ${ }^{16}$.

Los pacientes asintomáticos con hallazgo casual de esta fístula durante una laparotomía deberían ser tratados debido al riesgo de desarrollar colecistitis, colangitis y carcinoma de vesícula biliar ${ }^{17}$.

Consideramos este caso de gran interés por la manera atípica de presentación, en forma de hemorragia digestiva, en una paciente que carecía de los síntomas típicos de patología biliar (ictericia, dolor abdominal etc.), y que tampoco presentaba alteraciones analíticas destacables, salvo por la anemización secundaria al sangrado y una elevación de reactantes de fase aguda. 
Nuestra paciente presentaba importante comorbilidad, y en sus antecedentes ya constaba la patología biliar (colelitiasis y colecistitis crónica descritas en estudios radiológicos previos). En este caso, las exploraciones realizadas nos ayudaron a llegar al diagnóstico rápidamente, pero no siempre es así, por lo que es necesario tener en mente esta entidad a la hora de hacer diagnósticos diferenciales, especialmente en personas de edad avanzada o con importante comorbilidad y sobre todo si presentan diarrea crónica de etiología no filiada y antecedentes de enfermedad litiásica biliar.

\section{BIBLIOGRAFÍA}

1. Hession PR, Rawlinson J, Hall JR, Guyer PB. The clinical and radiological features of cholecystocolic fistulae. Br J Radiol 1996; 69: 804809.

2. Glen F, ReEd C, Grafe WR. Biliary enteric fistula. Surg Gynecol Obstet 1981; 153: 527-531.

3. Le Blanc KA, Barr LH, Rush BM. Spontaneous biliaryenteric fistulas. South Med J 1983; 76 : 1248-1252.

4. RAstogi R. Cholecystocolic fistula secondary to gallbladder carcinoma: a rare case. Saudi J Gastroenterol 2008; 14: 144-146.

5. Ahn SI, Hong KC, Hur YS, Lee KY, Kim SJ, Seo JM et al. Cholecystocolic fistula caused by blunt trauma. Injury 2001; 32: 341-342.

6. Costi R, Randone B, Violi V, Scatton O, Sarli L, Soubrane O et al. Cholecystocolonic fistula: facts and myths. A review of the 231 published cases. J Hepatobiliary Pancreat Surg 2009; 16: 8-18.

7. Savvidou S, Goulis J, Gantzarou A, Ilonidis G. Pneumobilia, chronic diarrea, vitamin $\mathrm{K}$ malabsortion: a pathognomonic triad for chole- cystocolonic fistulas. WORLD J GASTROENTEROL 2009; 15: 4077-4082.

8. LeE JW, KIM MY, KIM YJ, SuH CH. CT of acute lower GI bleeding in chronic cholecystitis concomitant pseudoaneurysm of cystic artery and cholecystocolonic fistula. Clin Radiol 2006: 61: 634-636.

9. Penschuck C, Federmann G. Life-threatening lower gastrointestinal bleeding caused by erosion of the cystic artery in gallbladdertransverse colon fistula. Chirurg 1991; 62: 895-896.

10. KAPLAN BJ. Massive lower gastrointestinal hemorrhage from cholecystocolic fistula. Dis Colon Rectum 1967: 10: 191-196.

11. ChAFFeE JS. Cholecystocolic fistula with large bowel hemorrage. Ann Surg 1960; 152: 901904.

12. Swinnen L, SAinte T. Colonic gallstone illeous. J Belge Radiol 1995; 77: 272-274.

13. Goldberg RI, Phillips RS, Barkin SJ. Spontaneous cholecystocolonic fistula treated by endoscopic sphincterotomy. Gastrointest Endosc 1988; 34: 55-56.

14. Toll EC, Kelly MD. Succesful management of cholecystocolic fistula by endoscopic retrograde cholangiapancreatography: a report of two cases. Hong Kong Med J 2010; 16: 406408.

15. Llach J, Bordas JM, Elizalde JL, Enrico C, Ginès A, Pellisé $M$ et al. Sphincterotomy in the treatment of biliary leakage. Hepatogastroenterology 2002; 49: 1496-1498.

16. Saito A, Takano M, Gunji N, Watanabe K, Ishihata R, Utsumi Y et al. [Xanthogranulomatous cholecystitis associated with a suspicious colonic fistula and its spontaneous resolution]. Nihon Shokakibyo Gakkai Zasshi 2009; 106 : 691-697.

17. Ahrendt SA, Pitt HA. Biliary tract. En: Sabiston textbook of surgery $\left(17^{\text {th }}\right.$. Edition). Vol 52; 2005: p. 1621. 